\title{
Optical Characterization of Quinoline Yellow Fluorochemosensors for Analyzing Its Photonics Applications With Synthesized Nano Particles
}

Dr Raveendra Melavanki ( $\sim$ melavankiphysics@gmail.com )

M S Ramaiah Institute of Technology Department of Physics https://orcid.org/0000-0002-2175-8430

Vijaya Kumar

M S Ramaiah Institute of Technology

N L Ramesh

M S Ramaiah Institute of Technology

Diksha Singh

M S Ramaiah Institute of Technology

Daruka Prasad B

B M S I T BENGLURU

Dhananjaya $\mathbf{N}$

IBS - Bengaluru

\section{Research Article}

Keywords: UV-Vis, SEM, photoluminescence, AFM, FTIR, XRD

Posted Date: January 3rd, 2022

DOI: https://doi.org/10.21203/rs.3.rs-1113299/v1

License: (1) This work is licensed under a Creative Commons Attribution 4.0 International License.

Read Full License 


\section{Abstract}

Quinoline Yellow (QY) with the scientific name [sodium 2-(2, 3-dihydro-1,3-dioxo-1H-inden-2-yl) quinoline6,8-disulfonate] (SQDS) is investigated for its sensing properties as fluorochemosensors and its NLO applications. Pure SQDS is doped with copper ferrite and cerium oxide nanoparticles and studied for changes in spectral results. Change in absorption spectrum is observed depending on the polarity of solvents. Intensity of fluorescence also varies with different type of solvents. Optical characterization for SQDS is carried out via various spectroscopic techniques including UV-VIS spectroscopy, FTIR spectroscopy, Scanning Electron Microscopy and Photo Luminescence (PL) spectroscopy. Optical parameters like extinction coefficient, refractive index and bandgap energy are determined from absorption spectrum for both solution and film samples. XRD characterization is also performed for QY and for nanoparticle doped QY. For investigating Non-Linear optical (NLO) application of QY, films are prepared and optical imaging is performed via Atomic Force Microscopy (AFM). Characterization results are analysed and predicted for application in non-linear optics.

\section{Introduction}

A molecular sensor is also called chemosensor. These are molecules which is used for detection of ions by interaction with an analyte. There are two main parts of a chemosensor, one is the receptor and other is reporter. The reporter gives out any change in signal caused when the guest is binding to the receptor. Fluorescent chemosensors use fluorescence as an output signal change to detect presence of ions. These sensors are considered powerful tool for detection because they are highly sensitive, simple and used as real-time situ imaging. Quinoline moieties are bioactive compounds. Quinoline derivatives are extensively studied because of their wide range of application which includes antibacterial, antiinflammatory, antifilarial, antifungal, local anaesthetic agents. Quinoline derivatives are found to have exceptional mechanical properties, which contributes to generation of high efficient materials in transportation of electrons. Characteristics such as good film formation, high electron mobility, great efficiency for photoluminescence and high thermal stability, makes quinoline derivatives to be of application in OLEDs .So, compounds containing quinoline owes to improved brightness and luminescence efficiencyin organic light emitting diodes[1-8],[18-26].

Organic crystals contains electron acceptor and donor groups on either side of conjugated $\pi$-bond. Mobility is increased as a result of overlapping between $\pi$ orbits and delocalized electronic charge. Increase in optical non-linearity happens when electron charge density distribution is enhanced in excited state or ground state. NLO applications of quinoline derivatives are significantly studied and investigated [9-13]. Ultrafast responses, low dielectric constant and broadband electronic responses are the key properties responsible for its non-linear optical properties (NLO). Quinoline moiety acts as electron acceptor and show high first order hyperpolarization. Quinoline based crystals that are optically nonlinear have been found to exhibit exceptionally high macro and microscopic non-linear optical susceptibility in addition to good environmental and thermal stability[14-17],[27]. 
In this present work, we investigate quinoline yellow derivative, SQDS [sodium 2-(2,3-dihydro-1,3-dioxo-1Hinden-2-yl) quinoline-6,8-disulfonate] with the help of spectroscopic methods namely UV-VISible spectroscopy and FTIR spectroscopy. XRD for pure SQDS is performed and is compared with XRD of mixed samples of SQDS with nanoparticles of copper ferrite and cerium oxide. Scanning electron microscopy is also performed for pure and nanoparticle doped SQDS. For investigating non-linear optical properties, films are made with solvent and SQDS and is compared using UV-Visbile and FTIR spectroscopic techniques. Film samples are tested under Atomic force microscope to study surface morphology and structure and uniformity of samples. Photoluminescence spectra is carried out for nanoparticle doped and pure powdered samples.

\section{Experimental Detail And Methods 2.1 Experimental details}

The quinoline derivative namely [sodium 2-(2, 3-dihydro-1,3-dioxo-1H-inden-2-yl) quinoline-6,8-disulfonate] was purchased and is strong yellow in color. The molecular structure, formula and molecular weight is illustrated in Fig. 1(a). With 95\% solubility in water, it can be considered as a potentially significant dye. Nano particles of copper ferrite and cerium oxide is synthesized in our lab and was used for doping the pure powder samples for spectroscopic analysis. Spectroscopy is done for the compound to understand and study its optical characteristics so as to identify its scope in luminescence applications. UV-Visible spectroscopy is carried out using Shimadzu UV-1800 and the solvent used was Dimethyl Sulfoxide (DMSO). The quinoline derivative SQDS is found to have complete solubility in DMSO and water. FTIR spectroscopy is one for sample in solution form and film form on Spectrum 3 FTIR spectrophotometer. XRD using D8 XRD and SEM using Tescan Vega 3 is carried out for powdered samples of pure quinoline and compared with mixture of pure quinoline in cerium oxide and copper ferrite. Film samples are prepared through drop casting method using DMSO as solvent. The concentration used for preparation of film samples is $0.1 \%$. Solution of SQDS in DMSO is heated on magnetic stirrer for 20 mins at 70 degree Celsius and $500 \mathrm{rpm}$. Then the solution is dropped on a clean glass slide and heated in oven at 50 degree Celsius so that DMSO can evaporate and we can get SQDS film. AFM is carried out for studying the surface morphology of film samples. Also, photoluminescence of pure SQDS and nanoparticle doped SQDS is analyzed using FP-8300 JARCO for PL spectra determination

\subsection{Synthesis of copper ferrite nanoparticles:}

Copper ferrite was prepared by solution combustion method using freshly prepared Oxalyl Dihydrazine $(\mathrm{ODH})$ as a fuel. Stochiometric calculation was adopted to balance the equation as mentioned below:$2.416 \mathrm{~g} \mathrm{Cu}(\mathrm{NO} 3) 2+8.09 \mathrm{~g} \mathrm{Fe}(\mathrm{NO}) 3+4.7238 \mathrm{~g} \mathrm{ODH}+25 \mathrm{ml}$ of double distilled water. was used to obtain CuFe2O4 compound. The precursors were mixed in the crystalline dish and subjected to magnetic stirring for 20 minutes. After the proper mixing, it is transferred to the Muffel furnace which is preheated at 400 ${ }^{\circ} \mathrm{C}$. within 20 minutes of time the smoldering flame was observed and burn completes the reaction. Here 
the ratio of reducing and oxidizing valencies were kept 1. Fig. 1(b) shows molecular structure for copper ferrite nanoparticles.

\subsection{Synthesis of cerous oxalate nanoparticles:}

Chemicals Required: Cerium (III) nitrate Hexahydrate (Ce(NO3)3.6H2O) (HiMedia-AR Grade), Oxalic acid Dihydrate (C2H2O4.2H2O) (SDFCl-AR Grade), Sodium Hydroxide (SRL), Distilled water were all used as received.

Preparation of the Cerous Oxalate Nanoparticles: (Sample No. 82) In a typical synthetic procedure, 0.03M of $\mathrm{Ce}(\mathrm{NO} 3) 3.6 \mathrm{H} 2 \mathrm{O}$ was dissolved in $20 \mathrm{ml}$ of Distilled water to form the transparent aqueous Cerium nitrate solution. With continuous magnetic stirring, $2 \mathrm{ml}$ of $0.365 \mathrm{M} \mathrm{NaOH}$ was added to the above solution which results in the pale white colour. $0.876 \mathrm{~g}$ of Oxalic acid was also added to the above solution resulting in the pale yellow colour ensuing $\mathrm{pH}=1$. After 2 minutes of stable suspension of the solution in the beaker, particles settled down resulting in the white precipitate. The obtained precipitate along with the supernatant solution was poured on the Watchglass and after two days, complete supernatant solution evaporated. After one month, about $80 \%$ of the powder was grown into crystal form [28-33]. Fig. 1(c) shows molecular structure for cerium oxide.

\subsection{Characterization techniques}

\subsubsection{UV-vis spectroscopy.}

UV-vis absorbance spectra in the wavelength range $200-800 \mathrm{~nm}$ were measured using UV-Vis spectrophotometer model Shimadzu UV-1800 in BMSIT, Bangalore.

\subsubsection{XRD.}

The crystal structures of the powder samples at a scanning rate of 0.02 per second in the range of 20 to 80 using D8 XRD model in CAMT department, MSRIT, Bangalore. Graphs and peak analysis was done using origin software.

\subsubsection{FT-IR.}

The IR absorption bands of samples in the range of 4000 to $400 \mathrm{~cm}^{-1}$ pellets were analyzed using using Spectrum 3 FTIR spectrograph in BMSIT, Bangalore.

\subsubsection{Photoluminescence Spectra}

Fluorescence is performed using Model name: FP-8300 with Xe lamp in medium sensitivity for the range 242-897 $\mathrm{nm}$ at Mysore University, Mysore.

\subsubsection{AFM and SEM}

Atomic force microscopy was performed for film samples at various inclinations in IISC, Bangalore. Scanning electron microscopy was performed for powdered samples in BMSCE, Bangalore. 


\section{Results And Discussion}

\subsection{UV-Visible Spectroscopy Analysis}

UV-Visible spectrum for solution sample and film sample of SQDS in solvent DMSO is shown in Fig. 2. Positive solvatochromism effect is observed in absorption peaks. Absorption peak for film sample shifts to longer wavelength region due to increase in solvent polarity. This phenomenon is also called red shift or bathochromic shift. Most of the DMSO has evaporated in film sample and hence a change in absorbance value is also observed. There is a hypochromic effect in film samples as compared to liquid solution samples. Hypochromic effect is the phenomenon when there is decrease in intensity of absorption. Optical density peaks are found at $354 \mathrm{~nm}$ for solution sample and at $452 \mathrm{~nm}$ for film samples.

\subsection{FTIR Spectroscopy Analysis}

FTIR results for SQDS in solution form and in film form are illustrated in Fig. 3(a) and Fig. 3(b) respectively. Infrared absorption spectrum is produced to identify chemical bonds in molecule. The obtained spectrum is a unique profile for every sample and consists of molecular fingerprint which can be used for identifying molecules in different samples. Two types of analysis can be done with FTIR spectrum- functional group region and fingerprint region. Below $1500 \mathrm{~cm}^{-1}$ in the obtained infrared spectrum gives the fingerprint of the sample. However it is not reliable for studying about the chemistry of molecules. Above $1500 \mathrm{~cm}^{-1}$ in the spectrum is significant as it gives information about functional groups present in the sample. Table 1 shows functional groups. 
Table 1

FTIR Functional groups

\begin{tabular}{|ll|}
\hline cm$^{-1}$ & Functional group \\
\hline 3696 & O-H (alcohol) \\
\hline 3465 & $\mathrm{~N}-\mathrm{H}$ (primary amine) \\
\hline 3384 & $\mathrm{~N}-\mathrm{H}$ (aliphatic primary amine) \\
\hline 2994 & $\mathrm{C}-\mathrm{H}$ (alkane) \\
\hline 2887 & $\mathrm{~N}-\mathrm{H}$ (amine salt) \\
\hline 2075 & $\mathrm{~N}=\mathrm{C}=\mathrm{S}$ (isothiocyanate) \\
\hline 1990 & $\mathrm{~N}=\mathrm{C}=\mathrm{S}$ (isothiocyanate) \\
\hline 1872 & $\mathrm{C}-\mathrm{H}$ (aromatic compound) \\
\hline 1656 & $\mathrm{C}-\mathrm{H}$ (aromatic compound) \\
\hline 1421 & $\mathrm{C}=\mathrm{C}$ (conjugated alkene) \\
\hline 1315 & $\mathrm{~S}=\mathrm{H}$ (carboxylic acid) \\
\hline 1035 & $\mathrm{C}-\mathrm{O}$ (vinyl ether) \\
\hline
\end{tabular}

\subsection{XRD Analysis}

XRD plots for pure SQDS and copper ferrite doped SQDS and pure SQDS and cerium oxide doped SQDS is shown in Fig. 4(a) and Fig. 4(b) respectively. Material is irradiated with X-rays and the intensities are recorded with respect to the scattering angle at which it leaves the sample. XRD peaks give information about the atomic distribution in unit cell and the XRD pattern provides information on defects and particle size. Samples are grinded into fine powder and then analyzed. Peak width is inversely proportional to crystal size. Sharp peaks corresponds to crystallinity and soft peaks represents amorphous form in the sample. Change in peaks due to doping represents parameter change in unit cell. XRD for copper ferrite is shown in Fig. 4(c).

\subsection{Photoluminescence Spectra Analysis}

Photoluminescence Spectra for pure SQDS, copper ferrite doped SQDS and cerium oxide doped SQDS is shown in Fig. 5(a), Fig. 5(b) and Fig. 5(c) respectively. Material under analysis is stimulated with photon of excitation wavelength of $354 \mathrm{~nm}$. This causes photo-excitation in the sample and in turn it causes electron to jump to higher state and then release photon to come back to ground state. This emission of light is called photoluminescence. Emission is monitored at a fixed excitation wavelength observed from absorption spectrum. The area under the curve gives value for bandgap energy. PL spectra for pure nano particle of copper ferrite and cerium oxide is shown in Fig. 5(d) and Fig. 5(e) respectively. 


\subsection{AFM Analysis}

AFM for film sample is shown in Fig. 6(a) and Fig. 6(b). AFM is a no-contact probing microscopy imaging technique with near atomic resolution to study surface topology. Surface roughness is quantified and uniformity of film samples is understood. Low rms is observed in film samples which shows uniformity of surface.

\subsection{SEM Analysis}

SEM for powdered samples of pure SQDS, copper ferrite doped SQDS and cerium oxide nanoparticle doped SQDS is illustrated in Fig. 7, Fig. 8 and Fig. 9. This imaging techniques is used for studying topology of surface for the samples. SEM uses electron to take images. Cross section of particles is observed whether the particle are porous, hollow or solid.

Absorption spectrum is used for determining optical parameters like extinction coefficient Fig. 10, refractive index Fig. 11 and band gap energies Fig. 12.

\subsection{Absorption and emission spectrum in different solvents}

Absorption and emission spectrum for Quinoline Yellow is tested in four different solvents namely water, ethanol, methanol and dimethylsulfoxide. With increase in solvent polarity, there is an observable red shift in absorption wavelength. This bathochromic shift in absorption is due to change in solvent polarities. With increase in polarity of solvent, absorption peaks tends to shift towards longer wavelength. Also, emission spectrum undergoes change due to solvent polarity. Fig. 13 and Fig. 14 shows solvent effect on absorbance and fluorescence of the compound.

\section{Conclusion}

With all the spectroscopic method for characterization of SQDS, it can be deduced that presence of solvatochromism reflects presence of auxochromes in sample. Red shift confirms auxochromes and that in turn leads to presence of conjugation in molecules. FTIR illustrated no dramatic introduction or elimination in transmission peaks which means it does not show significant changes in chemistry of samples when converted into films. XRD showed crystallinity of samples owing to sharp peaks and it could further be used for determining the unit cell lattice dimensions. PL spectra showing high intensity peaks make the sample to be a good volunteer for laser dyes. SEM also gives clear resolution images which shows good topology of surface. AFM confirms low roughness and uniformity in film sample. This makes it a good candidate for non-linear optical applications. Dependence of absorption and emission spectrum on solvent polarity confirms presence of fluorophores and auxochromes which can have a significant scope in luminescence applications.

\section{Declarations}




\section{Acknowledgement}

Authors Dr Raveendra Melavanki and Dr Vijaya Kumar are grateful to Dr N V R Naidu, Principal, Dr A Jagannatha Reddy, Head, Department of Physics and the management of M.S.R.I.T, Bangalore for their encouragement and providing necessary facilities. Authors also acknowledge Centre for Advanced Material Characterization Technology (CAMT) of Ramaiah Institute of Technology (RIT) for Characterization facility and one of the author Diksha Singh is thankful to M S R I T Alumni Association Bengaluru - 560054 research fellowship.

\section{Funding:}

The present work is a part of research funded by Alumni Association of Ramaiah institute of technology, Bengaluru.

\section{Conflict of Interest:}

The article entitled "Optical characterization of Quinoline yellow fluorochemosensors for analyzing its photonics applications with synthesized nano particles" submitted by Raveendra Melavanki et.al for publication in Inorganic Chemistry Communications is based on original work and results of all the experiments are carried out by all authors under my supervision. No part of the article has been previously submitted for publication in any journals.

\section{Ethics Approval/Declaration:}

Ethics of research has been followed and all the data results are reported transparently in the paper. All the authors have adhered to accepted standards for ethical research study.

\section{Consent to Participate:}

All the individual authors have given their consent to participate in the research work.

\section{Consent for Publication:}

All the authors have consented to participate in the study with the objective to publish the results of study by submitting research work paper in the journal.

\section{Data Availability:}

All data generated or analysed during this study are included in this published article. A graphical abstract of the data results are presented below: 


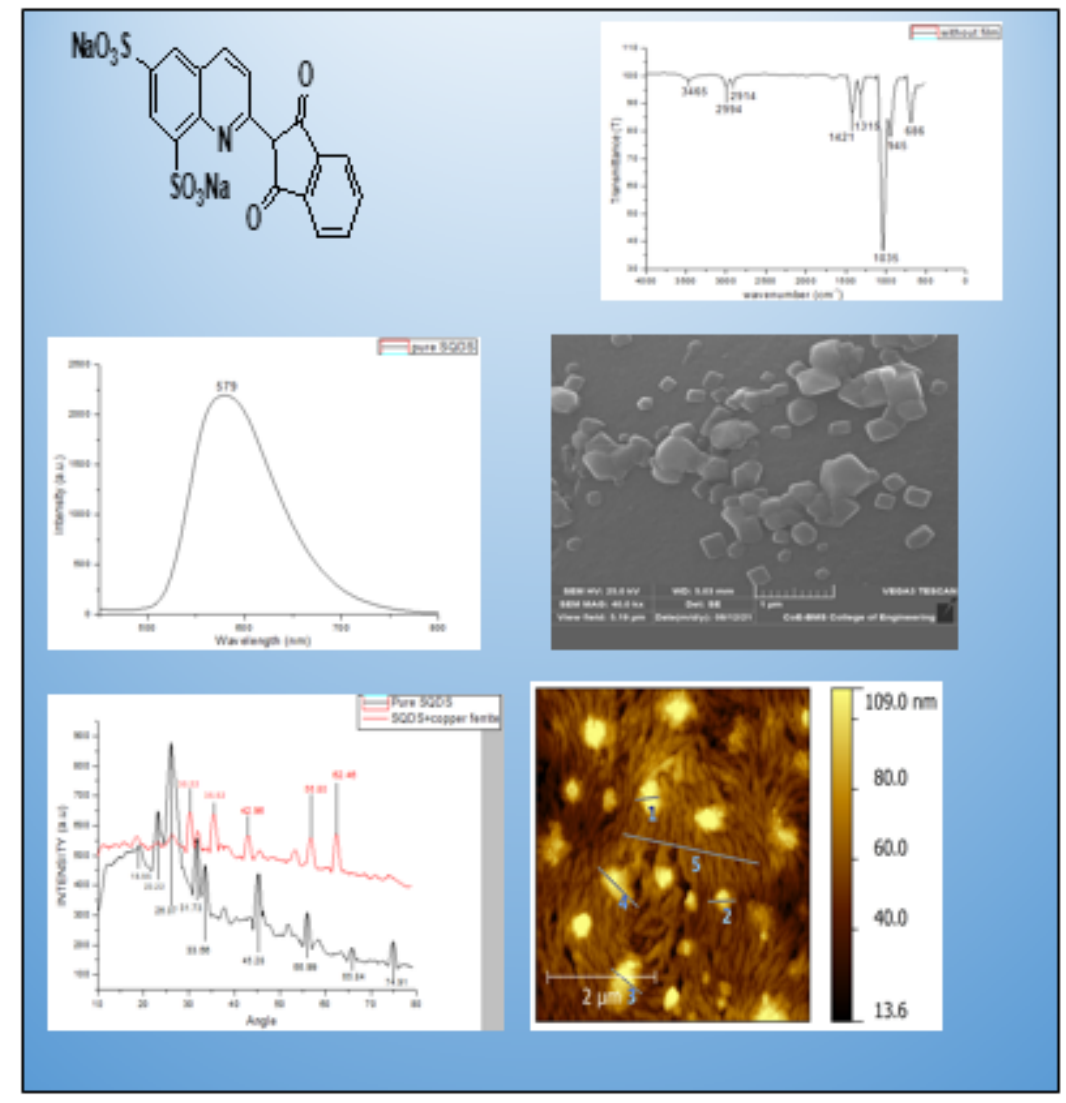

\section{Code Availability:}

Not Applicable

\section{Author's Contribution:}

Raveendra Melavanki: Guided the experimentation for study and read and reviewed the manuscript.

Vijaya Kumar: Conducted the UV-Visible spectroscopy and helped in the preparation of manuscript.

N L Ramesh: Did the XRD analysis and helped to prepare the manuscript.

Diksha Singh: Conducted the experimentation and analysed the experimental data and wrote the manuscript.

Daruka Prasad B: Synthesized nanoparticles of Cerium oxide and copper ferrite which was used for doping.

Dhananjay N: Helped in preparation of films and collection of FTIR data.

The manuscript was read by all individual authors.

\section{References}


1. E. Mikhailov, D.A. Svetlichnyi, O.N. Burov, Y.V Revinskii, G.A. Dushenko \& V.I. Minkin. Russian Journal of General Chemistry, 2015, 85, 1074-1077

2. Całus S, Gondek E, Danel A, Jarosz B \& Kityk A.V. Optics Communications, 2007, 271, 16-23

3. Qian Li, Zhaozhe Xu, Mingxin Yu, Hong Fan \& Bogeng Li. Journal of chemical research, 2011, 574578

4. Kumar Vinod, Gohain Mukut, Van Tonder, Johannes H, Ponra S, Bezuindenhoudt, B.C.B Ntwaeaborwa, O.M Swart, H.C. Optical Materials, 2015, 50, 275-281

5. Koścień, E. Gondek, M. Pokladko, B. Jarosz, R.O. Vlokh, A.V. Kityk. Materials Chemistry and Physics, 2009, 114, 860-867

6. M. Bahirwar, D.H. Gahane, R.G. Atram, S.V. Moharil. Physics Procedia, 2012, 29, 50-54

7. Hyong-Jun Kim, Journal of Luminescence, 2016, 176, 335-341

8. C. Dos Santos, R.O. Servilha, E.F. de Oliveira, F.C. Lavarda, V.F. Ximenes, L.C. da Silva-Filho. Journal of Fluorescence, 2017, 27, 1709-1720

9. M. Bahirwar, R.G. Atram, R.B. Pode, S.V. Moharil. Materails Chemistry and Physics, 2007, 106, 364368

10. Lewińska, K. Khachatryan, K.S. Danel, Z. Danel, J. Sanetra, K.W. Marszałek. Polymers, 2020, 12, 2707

11. Singh, V. Srivastava, M.A. Quraishi. Journal of Molecular Liquids, 2016, 216, 164-173

12. X Zhu, D Wang, H Huang, X Zhang, S Wang, R Liu, H Zhu. Dyes and Pigments, 2019, 171, 1-9

13. Karuppasamy et al. Journal of Molecular Structure, 2020, 1210, 128036

14. Slodek, M. Filapek, G. Szafraniec, I. Grudzka, W.A. Pisarski, J.G. Malecki, L. Zur, M. Grela, W.

Danikiewicz, S. Krompiec. European Journal of Organic Chemistry, 2014, 24, 5256 - 5264

15. C. Dos Santos, A. de Andrade Bartolomeu, V.F. Ximenes, L. C. da Silva -Filho. Journal of Fluorescence, 2017, 27, $271-280$

16. Karuppasamy, T. Kamalesh, K. Anitha, Muthu Senthil Pandian, P. Ramasamy, Sunil Verma. Journal of Molecular Structure, 2020, 1210, 128036

17. AAM Farag, S.A. Halim and N. Roushdy et al. Journal of Molecular Structure, 2020, 1221128868

18. S. Rane, M.A. Kazi, S.M. Bagul, D.P. Shelar, R.B. Toche, M.N. Jachak. Journal of Fluorescence, 2010, 20, 415-420

19. Khalid, M. Adeel, M.A. Ullah, M. Usman Khan, M.N. Tahir, A.A.C. Braga. Journal of Saudi Chemical Society, 2019, 23, 546-560

20. Barbieriková, M. Bella, J. Lietava, D. Dvoranová, A. Staško, T. Füzik, V. Milata, S. Jantová, V. Brezová. Journal of Photochemistry and Photobiology A: Chemistry, 2011, 224, 123-134

21. K. Ranjith, Y.S. Mary, C.Y. Panicker, P.L. Anto, S. Armaković, S.J. Armaković, R. Musiol, J. Jampilek, C. Van Alsenoy. Journal of Molecular Structure, 2017, 1135

22. M. Nagpure, M.M. Duvenhage, S.S. Pitale, O.M. Ntwaeaborwa, J.J. Terblans, H.C. Swart. J Fluoresc., 2012, 22, 1271-1279 
23. Boukabcha, A. Djafri, Y. Megrouss, Ö. Tamer, D. Avcı, M. Tuna, N. Dege, A. Chouaih, Y. Atalay, A. Djafri, F. Hamzaoui. Journal of Molecular Structure, 2019, 1194

24. Thirumurugan, P. Vadivel, A. Lalitha \& S. Lakshmanan. Synthetic Communications, 2020, 50, 831-839 25. Solanki \& K.Surati. Journal of Fluorescence, 2019, 29, 865-875

26. Kalpana Sharma, Raveendra Melavanki, Basappa C Yallur, Raviraj Kusanur, N R Patil, Vikas M Shelar, Diksha Singh, Jones Rosario, Qurban Hussaini, Mohan. A and Arun Varma Thampan. Macromolecular Symposia, 2020, 392, 2000165

27. Raveendra Melavanki, S Vaijayanthimala, Basappa Chanabasappa Yallur, Vikas M Shelar, Diksha singh, Kishor Kumar Sadasivuni and N R Patil. Luminescence, 2020, 1-10

28. Raveendra Melavanki, Kalpana Sharma, V.T. Muttannavar, Raviraj Kusanur, Kariyappa Katagi, Swarna M Patra, Siva Umapathy, Kishor Kumar Sadasivuni, Vikas M Shelar, Diksha Singh, NR Patil, Varsha V Koppal, Journal of Photochemistry \& Photobiology, A: Chemistry, 2021, 404, 112956

29. Jha, R. Patra, S. Ghosh, A.K. Ganguli. Journal of Materials Chemistry, 2012, 22(13), 6356

30. L. Pop, A. Mesaros, et al. Nanomaterials, 2020, 10, 1614

31. Mary C Minu, G Vimal, K.P. Mani, G. Jose, P.R. Biju, C. Joseph, N.V. Unnikrishnan, M.A. Ittyachen. Journal of Materials Research and Technology, 2016, 5, 268-274

32. P.Ghorpade, R.Hari Krishna, Raveendra .M. Melavanki, Vikas Dubey \& N.R.Patil. Optik - International Journal for Light and Electron Optics, 2020, 208, 164533

33. Santosh P. Ghorpade, Raveendra Melavanki \& N. R. Patil. Symp. 2020, 392, 1900155

34. Santosh P. Ghorpade, Nagaraju Kottam, Raveendra Melavanki \& N.R. Patil. RSC Advances 2020, 10, $21049-21056$

\section{Tables}

Table 1: FTIR Functional groups 


\begin{tabular}{ll}
$\mathbf{c m}^{-1}$ & Functional group \\
\hline 3696 & $\mathrm{O}-\mathrm{H}$ (alcohol) \\
\hline 3465 & $\mathrm{~N}-\mathrm{H}$ (primary amine) \\
\hline 3384 & $\mathrm{~N}-\mathrm{H}$ (aliphatic primary amine) \\
\hline 2994 & $\mathrm{C}-\mathrm{H}$ (alkane) \\
\hline 2887 & $\mathrm{~N}-\mathrm{H}$ (amine salt) \\
\hline 2075 & $\mathrm{~N}=\mathrm{C}=\mathrm{S}$ (isothiocyanate) \\
\hline 1990 & $\mathrm{~N}=\mathrm{C}=\mathrm{S}$ (isothiocyanate) \\
\hline 1872 & $\mathrm{C}-\mathrm{H}$ (aromatic compound) \\
\hline 1656 & $\mathrm{C}-\mathrm{H}$ (aromatic compound) \\
\hline 1630 & $\mathrm{C}=\mathrm{C}$ (conjugated alkene) \\
\hline 1421 & $\mathrm{O}-\mathrm{H}$ (carboxylic acid) \\
\hline 1315 & $\mathrm{~S}=\mathrm{O}$ (sulfoxide) \\
\hline 1035 & $\mathrm{C}-\mathrm{O}$ (vinyl ether) \\
\hline
\end{tabular}

Figures 

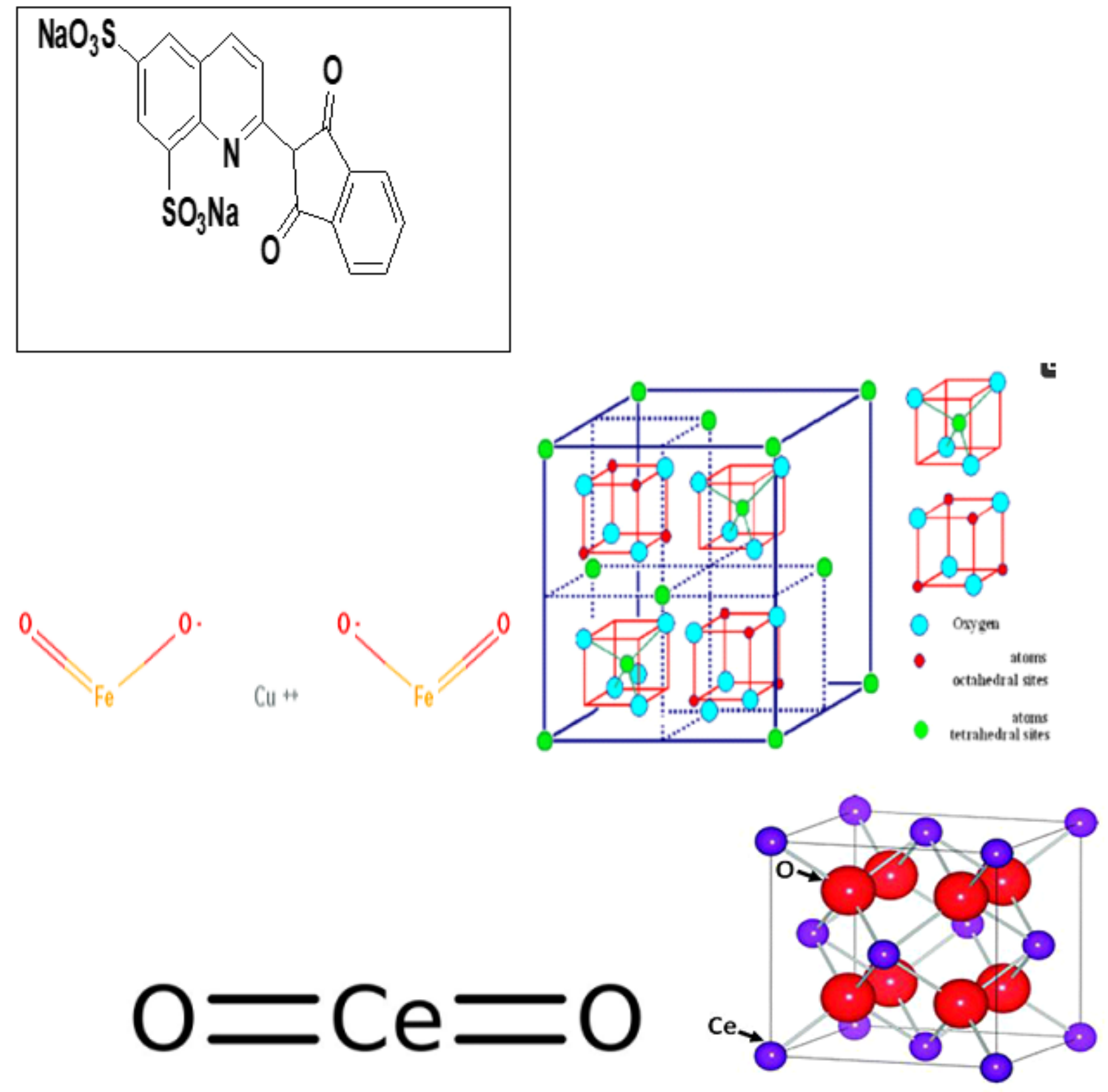

Figure 1

(a) Molecular structure for Quinoline Yellow SQDS

(b) Molecular structure and lattice structure for copper ferrite

(c) Molecular structure and lattice structure for cerium oxide 


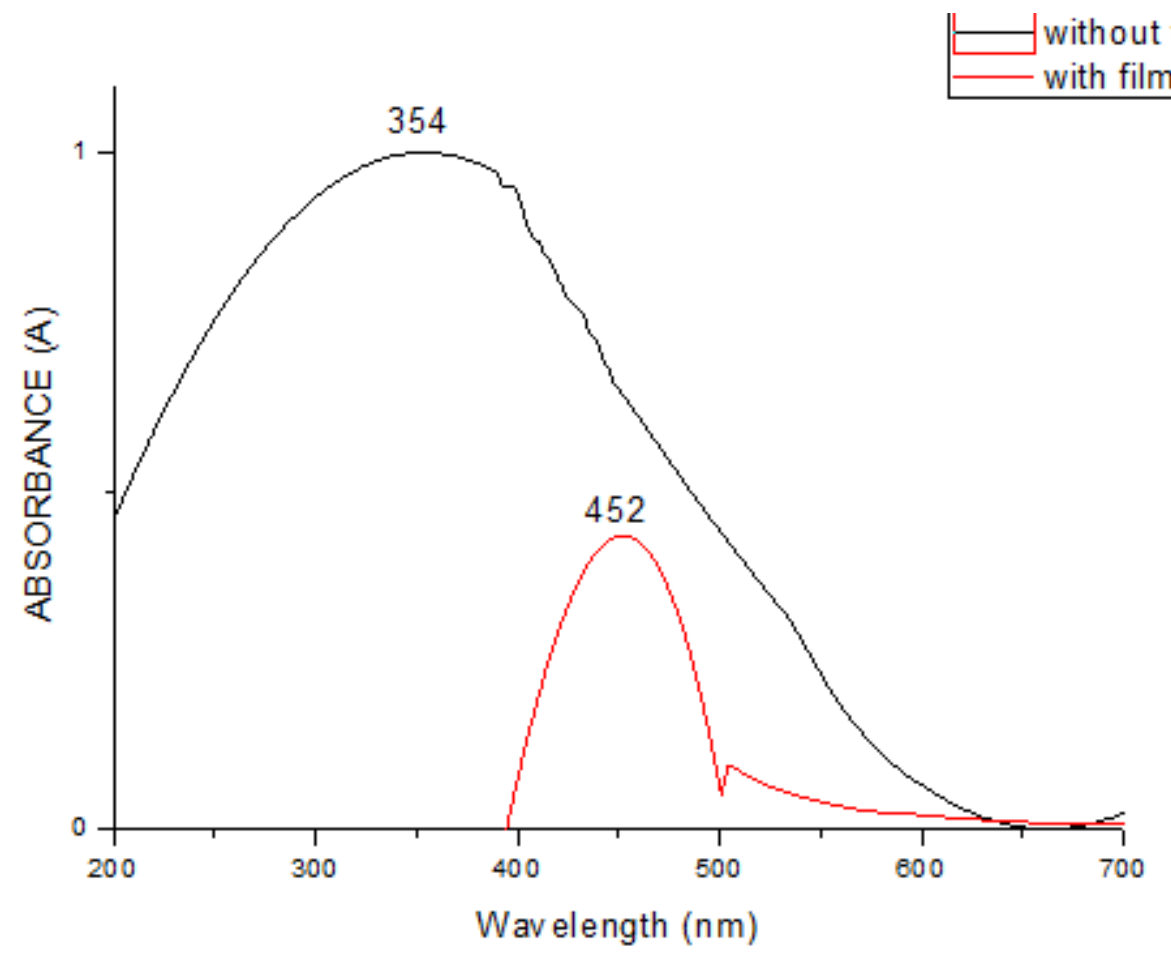

Figure 2

UV-Visible spectrum for SQDS with and without film
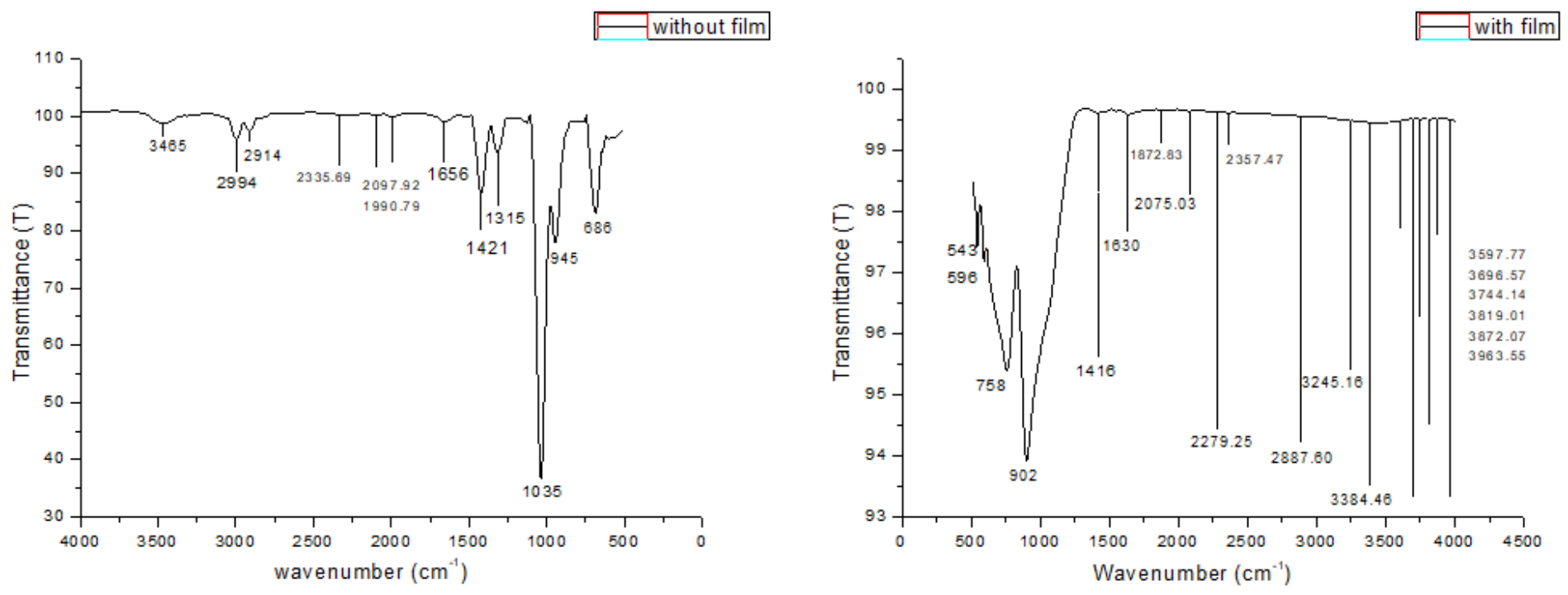

Figure 3

a FTIR spectrum for solution sample of SQDS in DMSO

b FTIR spectrum for film sample of SQDS 

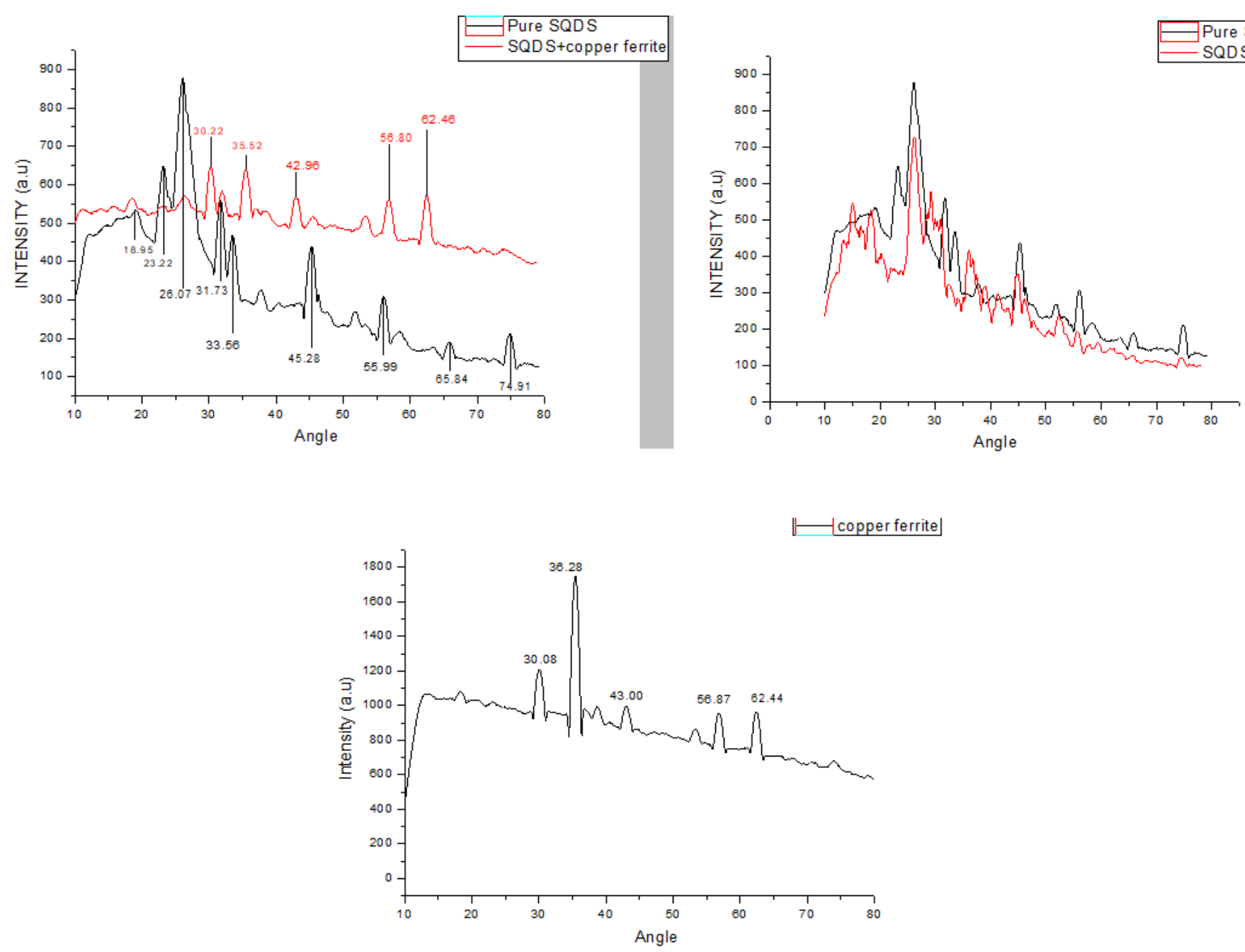

\section{Figure 4}

a XRD spectrum for quinoline derivative with Copper ferrite doping

b XRD spectrum for quinoline derivative with Cerium oxide doping

c XRD spectrum for pure copper ferrite 

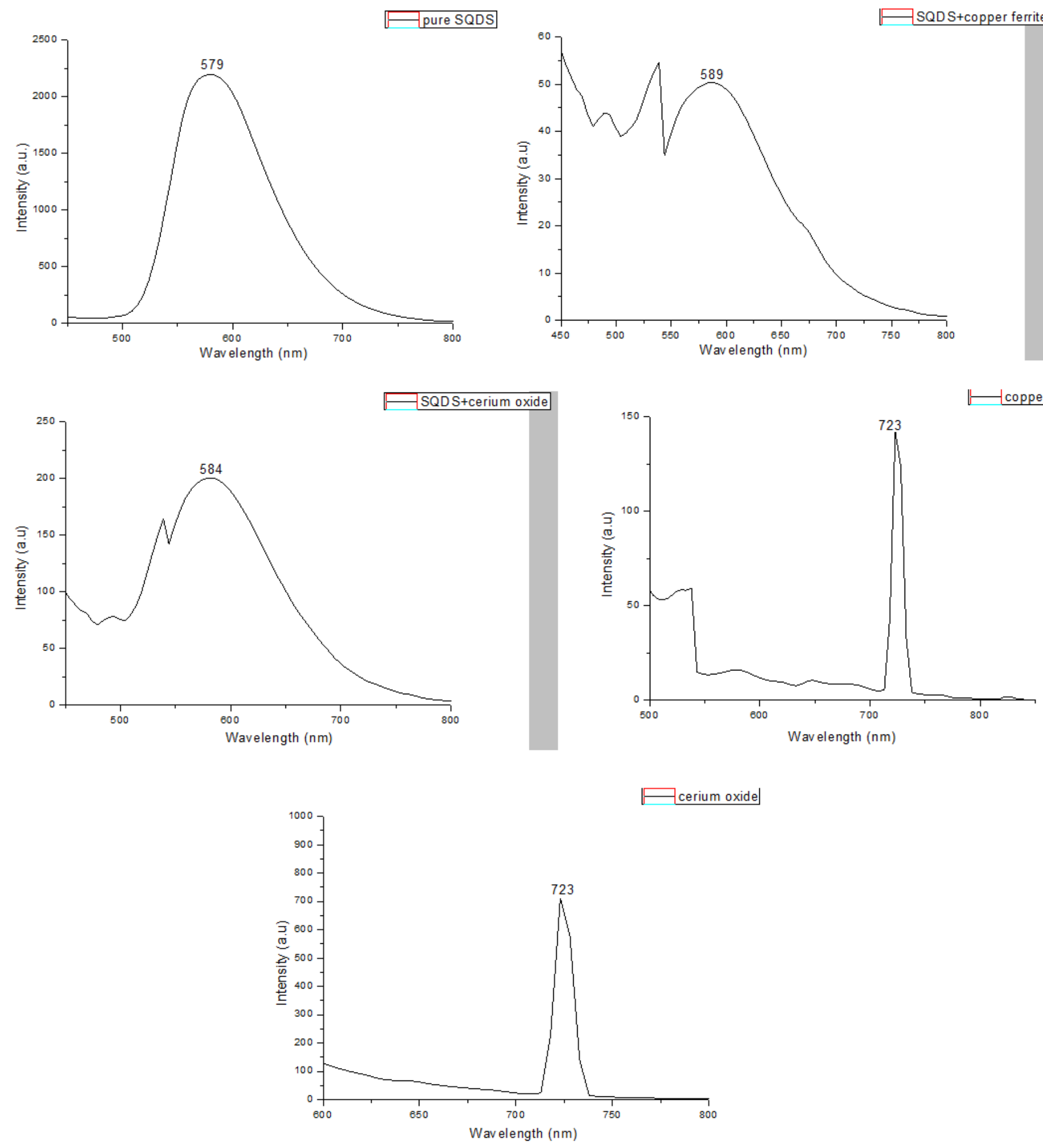

\section{Figure 5}

(a) PL spectra for pure SQDS

(b) PL spectra for SQDS doped with copper ferrite nanoparticles

(c) PL spectra for SQDS doped with cerium oxide nanoparticles 
(d) PL spectra for pure copper ferrite

(e) PL spectra for pure cerium oxide
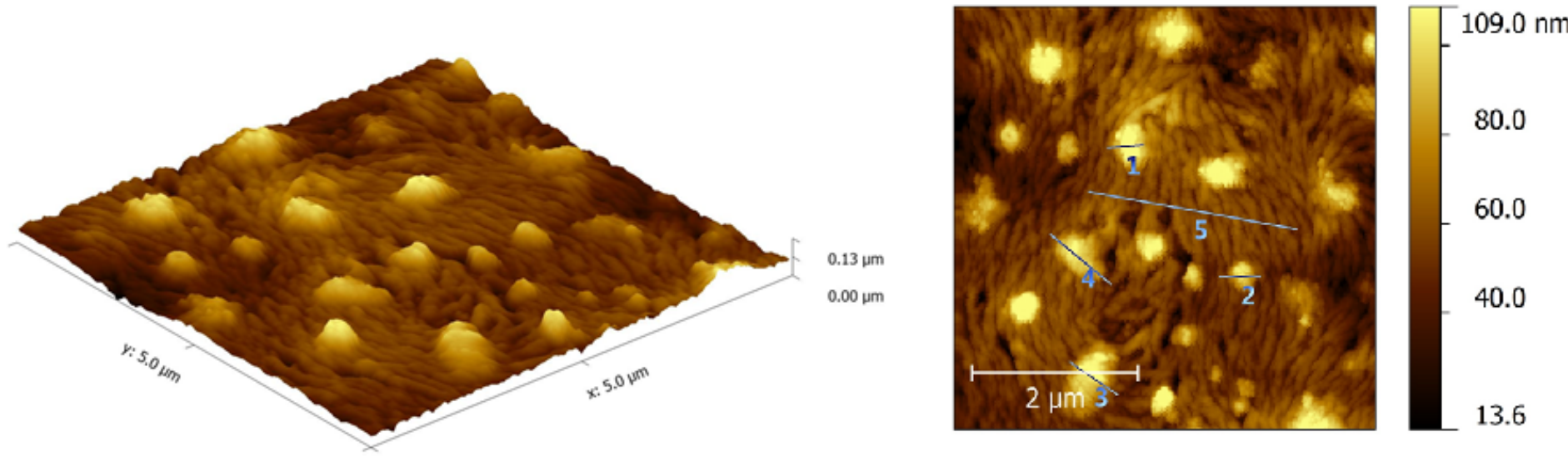

Figure 6

a AFM for SQDS film in 3D

b AFM for SQDS film for surface texture
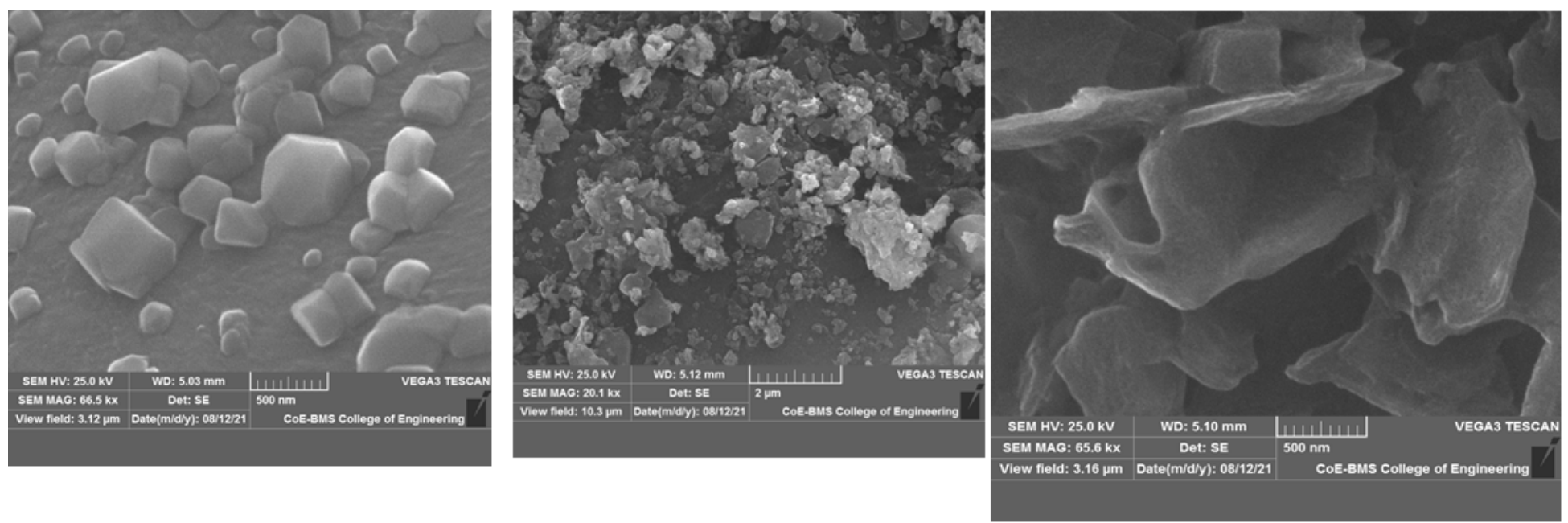

Figure 7

a SEM images for pure SQDS

b SEM images of SQDS doped with copper ferrite nanoparticles

c SEM image for SQDS doped with cerium oxide 


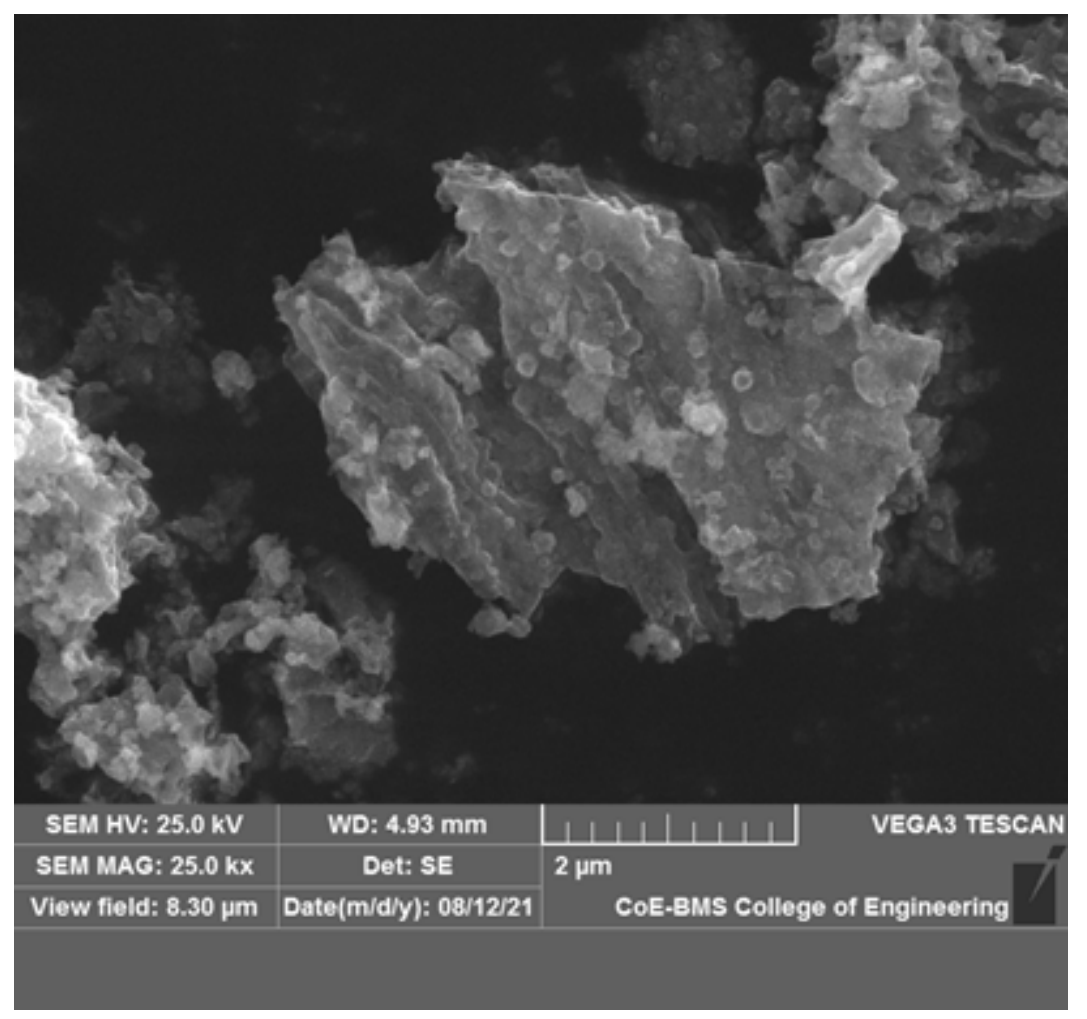

Figure 8

SEM image for pure copper ferrite

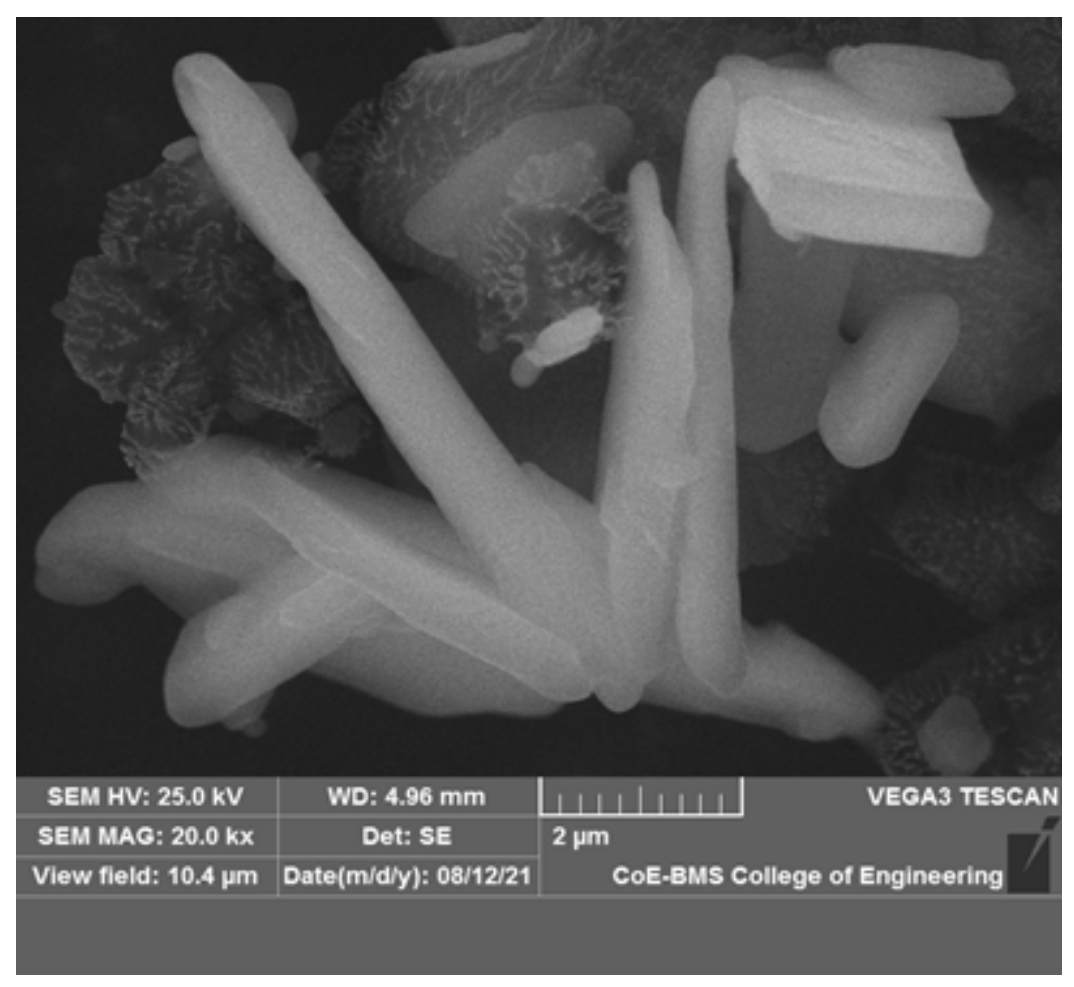

\section{Figure 9}

SEM image for pure cerium oxide 


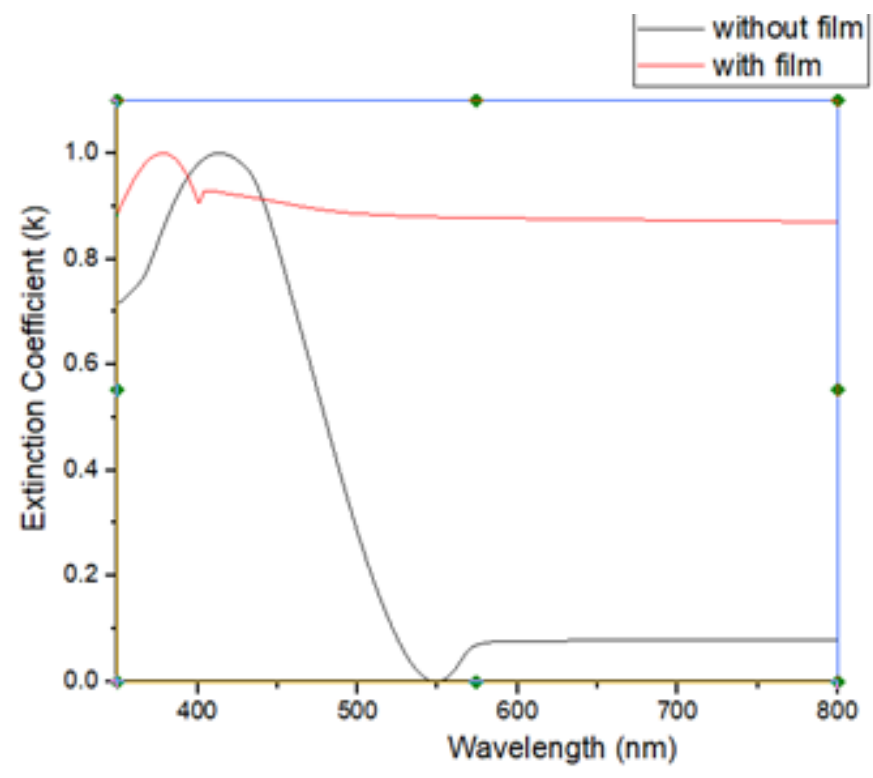

Figure 10

Extinction coefficient plot

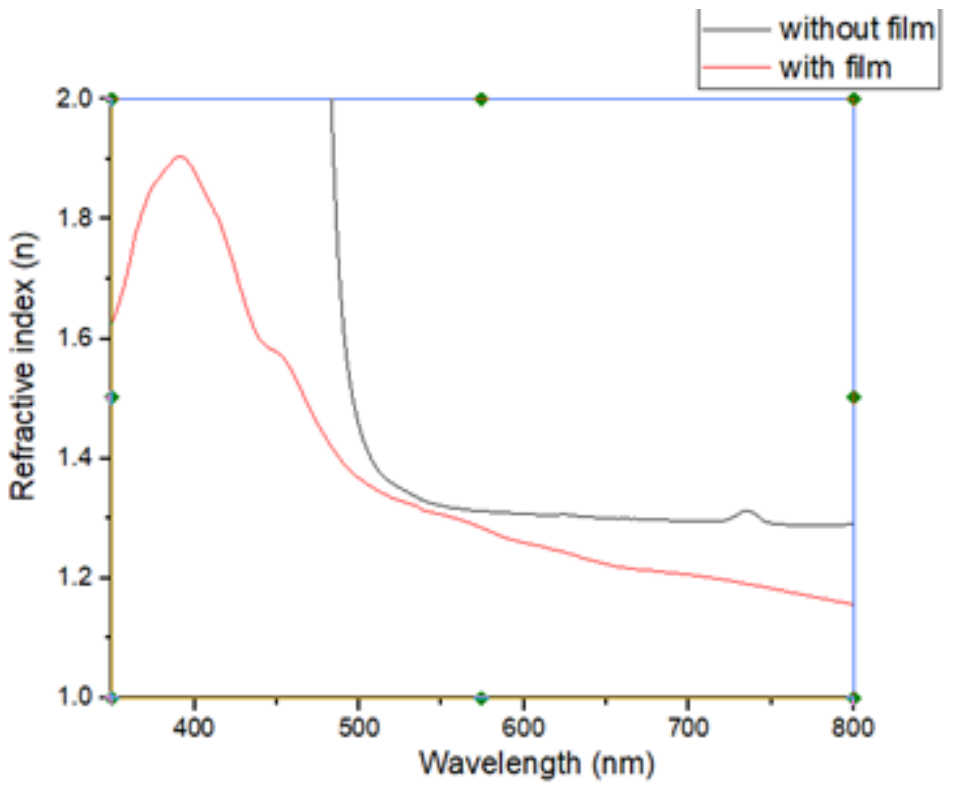

Figure 11

Refractive index plot 


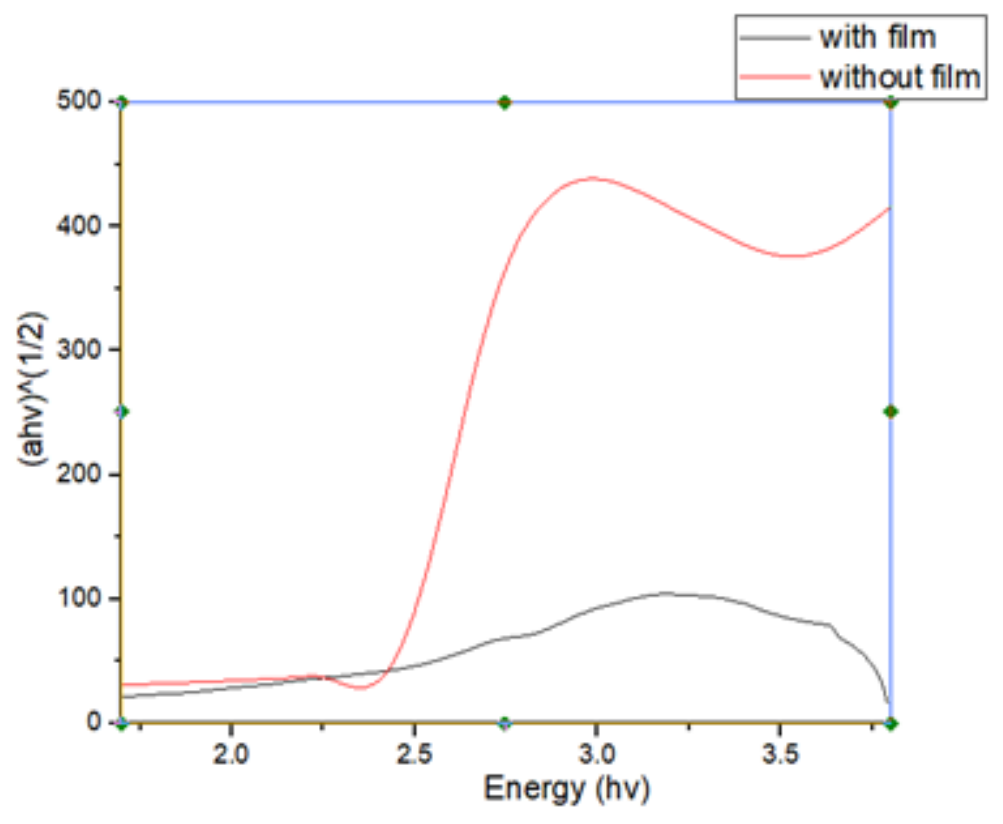

Figure 12

Bandgap energy Tauc plot

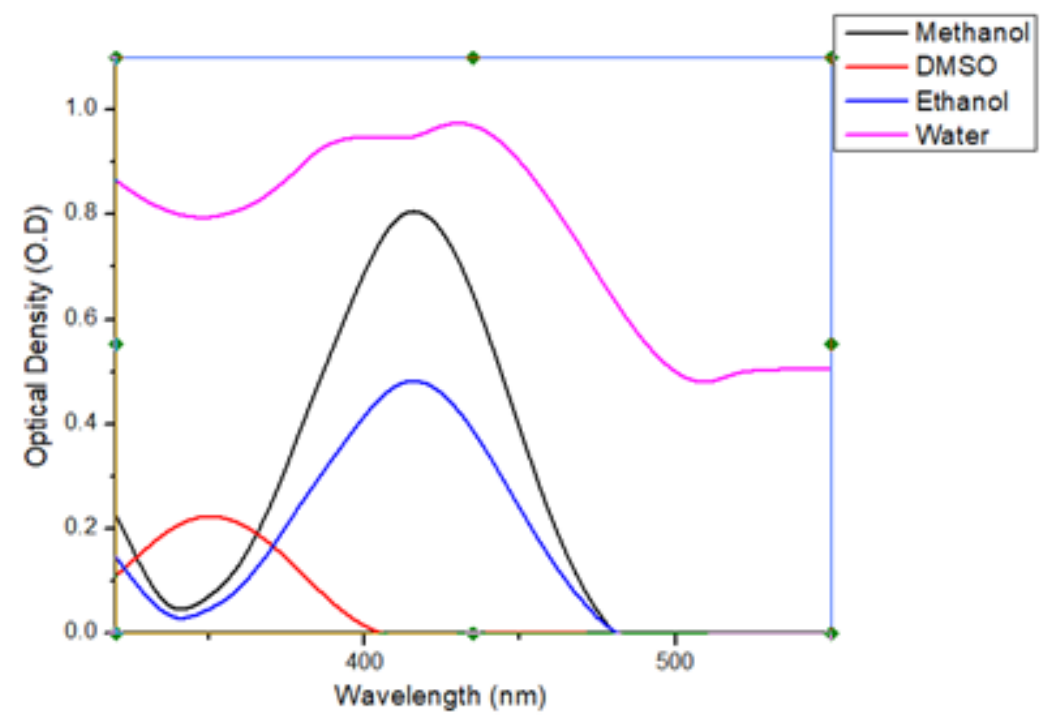

Figure 13

Solvent effect on Absorption spectrum 


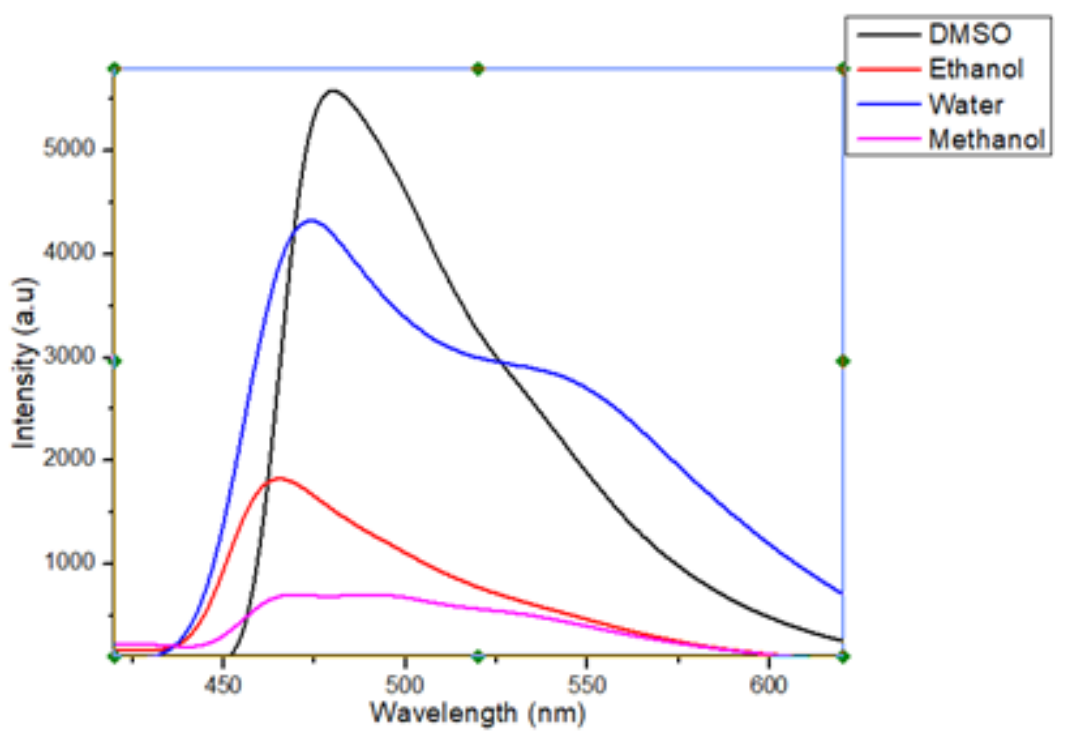

Figure 14

Solvent effect on fluorescence spectrum 